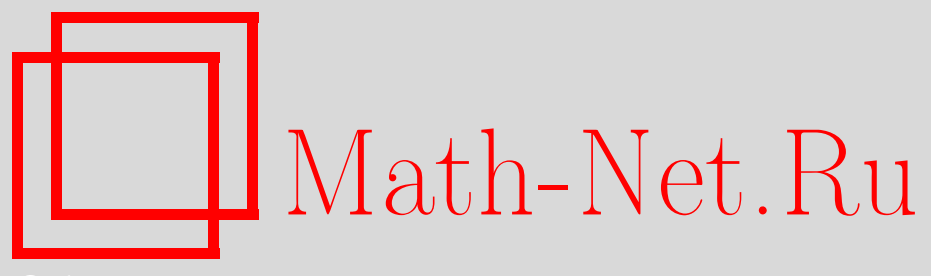

Б. Г. Конопельченко, Л. Мартинес Алонсо, Е. Медина, Интегрируемые квазиклассические деформации общих алгебраических кривых и соответствующие законы сохранения, ТМФ, 2007, том 151, номер 3, 458-469

DOI: https://doi.org/10.4213/tmf6059

Использование Общероссийского математического портала Math-Net.Ru подразумевает, что вы прочитали и согласны с пользовательским соглашением http://www . mathnet.ru/rus/agreement

Параметры загрузки:

IP: 18.208 .226 .222

26 апреля 2023 г., 02:40:26

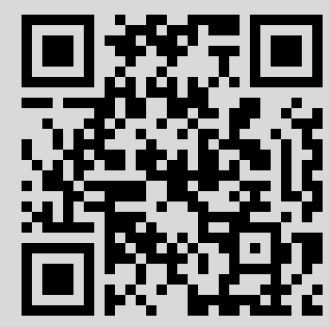




\title{
ИНТЕГРИРУЕМЫЕ КВАЗИКЛАССИЧЕСКИЕ ДЕФОРМАЦИИ ОБЩИХ АЛГЕБРАИЧЕСКИХ КРИВЫХ И СООТВЕТСТВУЮЩИЕ ЗАКОНЫ СОХРАНЕНИЯ
}

\begin{abstract}
Дана полная классификация интегрируемых деформаций общих алгебраических кривых, основанная на соотношениях Ленарда. Общее решение соотношения Ленарда строится из условия инвариантности относительно действия элемента группы Галуа кривой. Приведено несколько примеров, а также представлено несколько соответствующих законов сохранения.
\end{abstract}

Ключевые слова: алгебраические кривые, интегрируемые системы, соотношения Ленарда, законы сохранения.

\section{1. ВВЕДЕНИЕ}

Теория алгебраических кривых является фундаментальной составной частью методов анализа интегрируемых нелинейных дифференциальных уравнений [1]-[2]. Особый интерес представляет задача характеризации и классификации интегрируемых деформаций алгебраических кривых. В работах [2] Кричевер сформулировал общую теорию бездисперсионных иерархий интегрируемых моделей, связанных с деформациями алгебраических кривых, возникающими в методе усреднения Уизема. Другой подход к поиску интегрируемых деформаций алгебраических кривых $\mathcal{C}$, определенных алгебраическими уравнениями с единичным коэффициентом при старшей степени вида

$$
F(p, k):=p^{N}-\sum_{n=1}^{N} u_{n}(k) p^{N-n}=0, \quad u_{n} \in \mathbb{C}[k],
$$

${ }^{*}$ Dipartimento di Fisica, Universitá di Lecce and Sezione INFN, Lecce, Italy.

E-mail: konopel@le.infn.it

${ }^{\dagger}$ Departamento de Física Teórica II, Universidad Complutense, Madrid, Spain. E-mail: luism@fis.ucm.es

${ }^{\ddagger}$ Departamento de Matemáticas, Universidad de Cádiz, Puerto Real, Cádiz, Spain. E-mail: elena.medina@uca.es 
был предложен в работах [3]-[7]. Чтобы ввести такие деформации, нужно рассмотреть $N$ ветвей $p_{j}=p_{j}(k), j=1, \ldots, N$, многозначной функции, определенной формулой (1):

$$
F(p, k)=\prod_{j=1}^{N}\left(p-p_{j}(k)\right)=0, \quad \mathbf{p}(k)=\left(p_{1}(k), \ldots, p_{N}(k)\right)^{\mathrm{T}} .
$$

В терминах этих ветвей задача может быть сформулирована так: требуется найти деформации кривой $\mathcal{C}(x, t)$, согласующиеся со степенями полиномов $u_{n}$ и характеризующиеся наличием функции действия $\mathbf{S}=\mathbf{S}(k, x, t)$, которая удовлетворяет следующим условиям.

1. Функция $\mathbf{p}=\mathbf{p}(k)$ может быть представлена в виде

$$
\mathbf{p}=\mathbf{S}_{x} .
$$

2 . Функции $\mathbf{S}_{x}$ и $\mathbf{S}_{t}$ как функции переменной $p$ являются мероморфными на $\mathcal{C}(x, t)$ с полюсами только при $k=\infty$.

Из этих условий следует, что р удовлетворяет уравнению

$$
\partial_{t} \mathbf{p}=\partial_{x} \mathbf{Q}
$$

где $\mathbf{Q}:=\mathbf{S}_{t}=\left(Q_{1}, \ldots, Q_{N}\right)^{\mathrm{T}}$ задается равенствами [3], [8]

$$
Q_{j}=\sum_{r=1}^{N} a_{r}(k, x, t) p_{j}^{N-r}, \quad a_{r} \in \mathbb{C}[k], \quad j=1, \ldots, N,
$$

с $Q_{j} \in \mathbb{C}[k, p] / \mathcal{C}$. Далее нам потребуется важный результат, касающийся поведения ветвей $p_{j}(k)$. Пусть через $\mathbb{C}((\lambda))$ обозначено поле рядов Лорана по переменной $\lambda$, которые содержат не более конечного числа членов с положительными степенями. Тогда имеет место теорема Ньютона [9].

Теорема 1. Существует положительное иелое число l такое, что $N$ ветвей

$$
p_{j}(z):=\left.\left(p_{j}(k)\right)\right|_{k=z^{l}}, \quad j=1, \ldots, N,
$$

являются элементами поля $\mathbb{C}((z))$. Более того, если $F(p, k)$ есть неприводимый над полем $\mathbb{C}((k))$ полином, то $l_{0}=N$ есть наименьшее допустимое значение $l, u$ ветви $p_{j}(z)$ могут быть пронумерованы таким образом, что

$$
p_{j}(z)=p_{N}\left(\epsilon^{j} z\right), \quad \epsilon:=e^{2 \pi i / N} .
$$

Соглашение об обозначениях. Впредь для заданной алгебраической кривой $\mathcal{C}$ через $z$ мы будем обозначать переменную, связанную с наименьшим положительным числом $l_{0}$, для которого замена переменных $k=z^{l_{0}}$ влечет $p_{j} \in \mathbb{C}((z))$ для любого $j$. Будем называть $l_{0}$ показателем Ньютона кривой $\mathcal{C}$.

Уравнение (2) может быть переписано в терминах потенциалов $u_{n}, n=1,2, \ldots, N$, в виде [4], [5]

$$
\partial_{t} \mathbf{u}=J_{0} \mathbf{a}
$$


где $J_{0}-(N \times N)$-матричный дифференциальный оператор, элементы которого выражаются через потенциалы и их производные следующим образом:

$$
\begin{aligned}
\left(J_{0}\right)_{11}= & N \partial_{x}, \\
\left(J_{0}\right)_{i 1}= & (i-1) \mathcal{P}_{i-1} \partial_{x}-\sum_{l=2}^{i-1} u_{i-l} \mathcal{P}_{l-1} \partial_{x}-N u_{i-1} \partial_{x}, \quad i \neq 1, \\
\left(J_{0}\right)_{i j}= & (i+j-2) \mathcal{P}_{i+j-2} \partial_{x}+(j-1) \mathcal{P}_{i+j-2, x}- \\
& \quad-\sum_{k=1}^{i-1} u_{i-k}\left[(k+j-2) \mathcal{P}_{k+j-2} \partial_{x}+(j-1) \mathcal{P}_{k+j-2, x}\right], \quad i \neq 1, \quad j \neq 1 .
\end{aligned}
$$

Величины $\mathcal{P}_{s}$ представляют собой степенные суммы [10]:

$$
\mathcal{P}_{s}:=\frac{1}{s}\left(p_{1}^{s}+\cdots+p_{N}^{s}\right)=\sum_{1 \leqslant i \leqslant s}^{(s)} \frac{1}{i}\left(u_{1}+\cdots+u_{N}\right)^{i},
$$

где индекс $(s)$ над знаком суммы означает, что удерживаются лишь члены веса $s$.

Следующая задача состоит в определении выражений для а (в уравнении (4)), зависящих от $k$ и $\mathbf{u}$ так, чтобы поток (4) был согласован с полиномиальной зависимостью потенциалов $\mathbf{u}$ от переменной $k$. Другими словами, если $d_{n}:=\operatorname{degree}\left(u_{n}\right)$ суть степени коэффициентов $u_{n}$ как полиномов от $k$, то должны выполняться неравенства

$$
\operatorname{degree}\left(J_{0} \mathbf{a}\right)_{n} \leqslant d_{n}, \quad n=1, \ldots, N .
$$

Стратегия поиска согласованных деформаций [4], [5] заключается в том, чтобы использовать соотношения типа соотношений Ленарда

$$
J_{0} \mathbf{r}=0, \quad \mathbf{r}:=\left(r_{1}, \ldots, r_{N}\right)^{\mathrm{T}}, \quad r_{j} \in \mathbb{C}((k)),
$$

и взять $\mathbf{a}:=\mathbf{r}_{+}$, где $(\cdot)_{+}$и $(\cdot)_{-}$используются для обозначения частей с неотрицательными и отрицательными степенями по $k$, соответственно. Теперь из тождества $J_{0} \mathbf{a}=J_{0} \mathbf{r}_{+}=-J_{0} \mathbf{r}_{-}$ясно, что достаточное условие согласованности для системы (4) имеет вид

$$
\max _{m=1, \ldots, N}\left\{\operatorname{degree}\left(J_{0}\right)_{n m}\right\} \leqslant d_{n}+1, \quad n=1, \ldots, N .
$$

Налагая это условие, мы получаем достаточное условие согласованности, которое зависит лишь от кривой (1) и не зависит от того, какое частное решение соотношения Ленарда было использовано. Все решения системы (7) для случаев $N=2$ и $N=3$ были даны в работах [4], [6], а для случая $N=4$ - в работе [7]. Таким образом, было обнаружено, что согласованные степени таковы:

$$
\begin{array}{lllllll}
N=2: & d_{1} \leqslant d_{2}+1 & & & & & \\
N=3: & (0,0,1) & (0,1,0) & (0,1,1) & (0,1,2) & (1,0,0) & (1,0,1) \\
& (1,1,0) & (1,1,1) & (1,1,2) & (1,2,1) & (1,2,2) & (1,2,3) \\
N=4: & (0,0,0,1) & (0,0,1,0) & (0,0,1,1) & (0,1,0,0) & \\
& (0,1,0,1) & (0,1,1,0) & (0,1,1,1) & (0,1,1,2) .
\end{array}
$$


Также в работе [7] был доказан общий результат для случая $N \geqslant 5$. Он формулируется следующим образом.

Теорема 2. Для любого $N \in \mathbb{N}(N \geqslant 5)$ степени $\left(d_{1}, \ldots, d_{N}\right)$ удовлетворяют условию согласованности (7) тогда и толъко тогда, когда

$$
d_{j}=0, \quad j=1,2, \ldots, N-3, \quad d_{N-2}, d_{N-1}, d_{N} \leqslant 1 .
$$

Следующим шагом при классификации деформаций является определение решений $\mathbf{r}$ соотношения Ленарда (6). В работах [4], [5] было доказано, что решение уравнения $J_{0} \mathbf{r}=0$ дается формулами

$$
\mathbf{r}=T \nabla_{\mathbf{u}} R, \quad R=\sum_{j=1}^{N} g_{j}(z) p_{j}, \quad \nabla_{\mathbf{u}} R=\left(\frac{\partial R}{\partial u_{1}}, \ldots, \frac{\partial R}{\partial u_{N}}\right)^{\mathrm{T}},
$$

где

$$
T:=\left(\begin{array}{cccc}
1 & -u_{1} & \cdots & -u_{N-1} \\
0 & 1 & \cdots & -u_{N-2} \\
\vdots & \vdots & \ddots & \vdots \\
0 & 0 & \cdots & 1
\end{array}\right)
$$

и $g_{j} \in \mathbb{C}((z))$. Таким образом, остается задача определения подходящего способа выбора функций $g_{j}$, при котором $R \in \mathbb{C}((k))$ и, следовательно, $\mathbf{r} \in \mathbb{C}((k))$. Эта задача рассматривалась в работе [6] в терминах элемента $\sigma_{0}$ группы Галуа кривой:

$$
\sigma_{0}\left(p_{j}\right)(z):=p_{j}\left(\epsilon_{0} z\right), \quad \epsilon_{0}:=e^{2 \pi i / l_{0}} .
$$

Таким образом, ясно, что требование $R \in \mathbb{C}((k))$ эквивалентно инвариантности функции $R$ относительно действия элемента группы $\sigma_{0}$ :

$$
R\left(\epsilon_{0} z, \sigma_{0} \mathbf{p}\right)=R(z, \mathbf{p})
$$

В настоящей работе мы сконцентрируем внимание на построении функций $R$ вида (10), удовлетворяющих условию инвариантности (12). Используя эти функции, мы сможем представить некоторые примеры интегрируемых систем деформаций. Чтобы определить инвариантные функции $R(10)-(12)$, необходимо классифицировать все согласованные степени потенциалов $(8),(9)$ в соответствии с $\sigma_{0}$ и $l_{0}$.

Работа организована следующим образом. Раздел 2 посвящен вычислению показателя Ньютона $l_{0}$ и элемента $\sigma_{0}(11)$ группы Галуа кривой в каждом из согласованных случаев (8) и (9). В разделе 3 мы рассматриваем задачу построения функции $R$ вида (10), удовлетворяющую условию (12), в каждом из согласованных случаев. Наконец, в разделе 4 все полученные результаты иллюстрируются некоторыми примерами интегрируемых деформаций алгебраических кривых, в которых мы также получаем некоторые законы сохранения, связанные с каждой интегрируемой деформацией. 
ТАБлицА 1. Значения $\sigma_{0}$ и $l_{0}$ для $N=2$.

\begin{tabular}{|c|c|c|}
\hline$\sigma_{0}$ & $l_{0}$ & $\left(d_{1}, d_{2}\right)$ \\
\hline$\left(\begin{array}{ll}p_{1} & p_{2} \\
p_{2} & p_{1}\end{array}\right)$ & 2 & $d_{2}>2 d_{1}, d_{2}$ нечетная \\
\hline$\left(\begin{array}{ll}p_{1} & p_{2} \\
p_{1} & p_{2}\end{array}\right)$ & 1 & $d_{2}<2 d_{1}$ или $d_{2}$ четная \\
\hline
\end{tabular}

ТАБлИЦА 2. Значения $\sigma_{0}$ и $l_{0}$ для $N=3$.

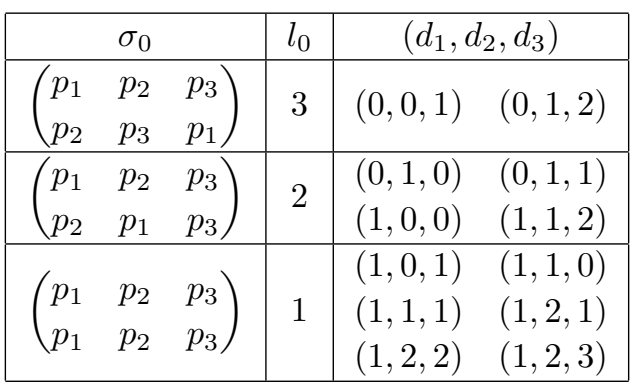

\section{2. ПОКАЗАТЕЛИ НЬЮТОНА}

\section{И ЭЛЕМЕНТЫ ГРУППЫ ГАЛУА КРИВОЙ}

Нашей ближайшей целью является классификация всех согласованных случаев в терминах соответствующего показателя Ньютона и элемента $\sigma_{0}(11)$ группы Галуа кривой. Прежде всего, заметим, что при $N=2,3,4$ ветви могут быть выражены через потенциалы, следовательно, используя разложения ветвей по степеням $z$ и определение (11), мы немедленно приходим к результатам, представленным в следующих таблицах.

В общем случае при $N \geqslant 5$ в силу теоремы Абеля ветви не могут быть записаны через потенциалы. Тем не менее, все алгебраические кривые, соответствующие (9),

ТАБлицА 3 . Значения $\sigma_{0}$ и $l_{0}$ для $N=4$.

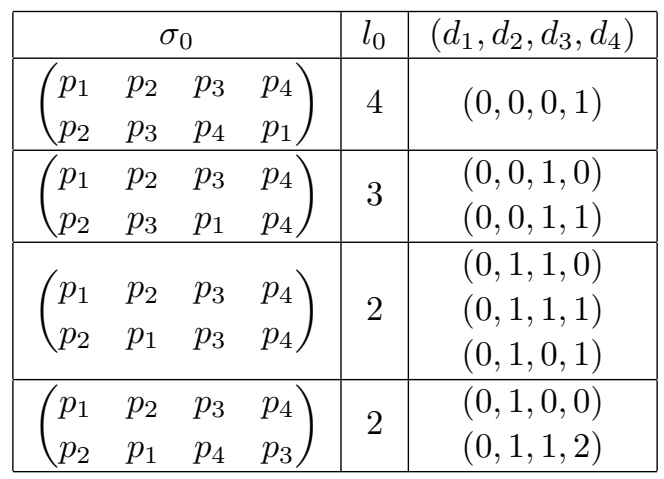


являются рациональными ( $k$ есть рациональная функция $p)$. Таким образом, классификация может быть проведена путем исследования асимптотического поведения $N$ ветвей $p_{j}, j=1,2, \ldots, N$, при $k \rightarrow \infty$. Для этого запишем потенциалы в виде

$$
u_{n}=\sum_{j=0}^{d_{n}} u_{n j} k^{j}
$$

Тогда имеем следующую классификацию.

- Пусть $\left(d_{1}, \ldots, d_{N-3}, d_{N-2}, d_{N-1}, d_{N}\right)=(0, \ldots, 0,0,0,1)$. В этом случае формула (1) может быть записана в виде

$$
k=\frac{1}{u_{N 1}}\left(p^{N}-\sum_{l=1}^{N} u_{l 0} p^{N-l}\right),
$$

так что

$$
p_{j}^{N} \sim u_{N 1} k \quad \text { при } \quad k \rightarrow \infty, \quad j=1,2, \ldots, N .
$$

Следовательно, $p_{j} \in \mathbb{C}\left(\left(k^{1 / N}\right)\right), j=1,2, \ldots, N$, и

$$
l_{0}=N, \quad \sigma_{0}=\left(\begin{array}{ccccc}
p_{1} & p_{2} & \cdots & p_{N-1} & p_{N} \\
p_{2} & p_{3} & \cdots & p_{N} & p_{1}
\end{array}\right) .
$$

- Пусть $\left(d_{1}, \ldots, d_{N-3}, d_{N-2}, d_{N-1}, d_{N}\right)=(0, \ldots, 0,0,1,0)$. Теперь формула (1) имеет вид

$$
k=\frac{1}{u_{N-11}}\left(p^{N-1}-\sum_{l=1}^{N} u_{l 0} p^{N-l-1}-\frac{u_{N 0}}{p}\right) .
$$

Таким образом, корни удовлетворяют соотношениям

$$
\begin{array}{rlrl}
p_{j}^{N-1} & \sim u_{N-11} k & \text { при } & k \rightarrow \infty, \quad j=1,2, \ldots, N-1, \\
p_{N} & \sim-\frac{u_{N 0}}{u_{N-11}} \frac{1}{k} \quad \text { при } \quad k \rightarrow \infty, &
\end{array}
$$

и мы имеем

$$
l_{0}=N-1, \quad \sigma_{0}=\left(\begin{array}{ccccc}
p_{1} & p_{2} & \cdots & p_{N-1} & p_{N} \\
p_{2} & p_{3} & \cdots & p_{1} & p_{N}
\end{array}\right) .
$$

- Пусть $\left(d_{1}, \ldots, d_{N-3}, d_{N-2}, d_{N-1}, d_{N}\right)=(0, \ldots, 0,1,0,0)$. Из уравнения (1) кривой следует, что

$$
k=\frac{1}{u_{N-21}}\left(p^{N-2}-\sum_{l=1}^{N-2} u_{l 0} p^{N-l-2}+\frac{u_{N-10}}{p}+\frac{u_{N 0}}{p^{2}}\right) .
$$

В таком случае

$$
\begin{aligned}
& p_{j}^{N-2} \sim u_{N-21} k \quad \text { при } \quad k \rightarrow \infty, \quad j=1,2, \ldots, N-2, \\
& p_{j}^{2} \sim \frac{u_{N 0}}{u_{N-21}} \frac{1}{k} \quad \text { при } \quad k \rightarrow \infty, \quad j=N-1, N .
\end{aligned}
$$


ТАБлицА 4. Классификация (9) по $\sigma_{0}$ и $l_{0}$.

\begin{tabular}{|c|c|c|c|c|c|c|}
\hline \multicolumn{4}{|c|}{$\sigma_{0}$} & \multirow{2}{*}{\multicolumn{2}{|c|}{$\begin{array}{l}l_{0} \\
N\end{array}$}} & \multirow{2}{*}{$\frac{\left(d_{1}, \ldots, d_{N}\right)}{(0, \ldots, 0,0,0,1)}$} \\
\hline$\left(\begin{array}{l}p_{1} \\
p_{2}\end{array}\right)$ & $\begin{array}{l}p_{2} \\
p_{3}\end{array}$ & $\begin{array}{ll}\cdots & 1 \\
\ldots & \end{array}$ & $\left.\begin{array}{cc}p_{N-1} & p_{N} \\
p_{N} & p_{1}\end{array}\right)$ & & & \\
\hline & $\begin{array}{l}p_{2} \\
p_{3}\end{array}$ & $\begin{array}{ll}\cdots & 1 \\
\ldots & \\
\end{array}$ & $\left.\begin{array}{cc}p_{N-1} & p_{N} \\
p_{1} & p_{N}\end{array}\right)$ & & $N-1$ & $\begin{array}{l}(0, \ldots, 0,0,1,0) \\
(0, \ldots, 0,0,1,1)\end{array}$ \\
\hline$\left(\begin{array}{l}p_{1} \\
p_{2}\end{array}\right)$ & $\ldots$ & $\begin{array}{c}p_{N-2} \\
p_{1}\end{array}$ & $\left.\begin{array}{ll}p_{N-1} & p_{N} \\
p_{N-1} & p_{N}\end{array}\right)$ & & $N-2$ & $\begin{array}{r}(0, \ldots, 0,1,1,0) \\
(0, \ldots, 0,1,1,1) \\
(0, \ldots, 0,1,0,1) \\
\end{array}$ \\
\hline$\left(\begin{array}{l}p_{1} \\
p_{2}\end{array}\right)$ & $\ldots$ & $\begin{array}{c}p_{N-2} \\
p_{1}\end{array}$ & $\left.\begin{array}{cc}p_{N-1} & p_{N} \\
p_{N} & p_{N-1}\end{array}\right)$ & $\begin{array}{l}N-2, \\
2(N-2),\end{array}$ & $\begin{array}{l}\text { если } N \text { четное } \\
\text { если } N \text { нечетное }\end{array}$ & $(0, \ldots, 0,1,0,0)$ \\
\hline
\end{tabular}

Таким образом, показатель Ньютона равен

$$
l_{0}=\left\{\begin{array}{llll}
N-2, & \text { если } & N & \text { четное } \\
2(N-2), & \text { если } & N & \text { нечетное }
\end{array}\right.
$$

а соответствующий элемент группы Галуа задается выражением

$$
\sigma_{0}=\left(\begin{array}{cccccc}
p_{1} & p_{2} & \cdots & p_{N-2} & p_{N-1} & p_{N} \\
p_{2} & p_{3} & \cdots & p_{1} & p_{N} & p_{N-1}
\end{array}\right)
$$

Действуя аналогичным образом в оставшихся случаях, получаем классификацию, отраженную в табл. 4 .

\section{3. ИНВАРИАНТНАЯ ФУНКЦИЯ $R$}

Чтобы получить иерархию интегрируемых деформаций (4), требуется определить функцию $R$ вида (10), удовлетворяющую условию инвариантности (12). Ввиду (12) различные случаи классифицируются по соответствующему элементу $\sigma_{0}$ группы Галуа кривой.

- Пусть $\sigma_{0}=\left(\begin{array}{ccccc}p_{1} & p_{2} & \cdots & p_{N-1} & p_{N} \\ p_{2} & p_{3} & \ldots & p_{N} & p_{1}\end{array}\right)$.

В соответствии с табл. 1-4 имеем $l_{0}=N, \quad\left(\epsilon_{0}=\epsilon=e^{2 \pi i / N}\right)$. Чтобы определить инвариантную функцию $R$, будем искать функции

$$
R_{k}=\sum_{j=1}^{N} \alpha_{j} p_{j} \quad \text { такие, что } \quad \sigma_{0}\left(R_{k}\right)=\epsilon_{0}^{-k} R_{k}, \quad k=0,1, \ldots, N-1 .
$$

Легко проверить, что

$$
\sigma_{0}\left(R_{k}\right)=\alpha_{N} p_{1}+\sum_{j=2}^{N} \alpha_{j-1} p_{j}
$$

так что из условия $\sigma_{0}\left(R_{k}\right)=\epsilon_{0}^{-k} R_{k}$ следует

$$
\alpha_{j-1}=\epsilon_{0}^{-k} \alpha_{j}, \quad \alpha_{N}=\epsilon_{0}^{-k} \alpha_{1} ; \quad j=2, \ldots N-1, N .
$$


Эта система допускает нетривиальные решения $\alpha_{j}=\epsilon_{0}^{j k} \alpha_{N}$. Таким образом, функция $R$ вида (10), удовлетворяющая условию (12), может быть записана следующим образом:

$$
R=\sum_{k=0}^{N-1} z^{k} f_{k}\left(z^{N}\right) \sum_{j=1}^{N} \epsilon_{0}^{j k} p_{j}
$$

где $f_{k} \in \mathbb{C}\left(\left(z^{N}\right)\right), k=0,1, \ldots, N-1$.

- Пусть $\sigma_{0}=\left(\begin{array}{ccccc}p_{1} & \cdots & p_{N-2} & p_{N-1} & p_{N} \\ p_{2} & \cdots & p_{N-1} & p_{1} & p_{N}\end{array}\right)$.

Соответствующий показатель Ньютона равен $l_{0}=N-1\left(\epsilon_{0}=e^{2 \pi i /(N-1)}\right)$. В этом случае $\sigma_{0}\left(p_{N}\right)=p_{N}$ или, что эквивалентно, $p_{N} \in \mathbb{C}((k))$. Поступим так же, как и в предыдущем случае, и будем искать функции

$$
R_{k}=\sum_{j=1}^{N-1} \alpha_{j} p_{j} \quad \text { такие, что } \quad \sigma_{0}\left(R_{k}\right)=\epsilon_{0}^{-k} R_{k}, \quad k=0,1, \ldots, N-2 .
$$

Поскольку действие элемента $\sigma_{0}$ на функцию $R_{k}$ задается выражением

$$
\sigma_{0}\left(R_{k}\right)=\alpha_{N-1} p_{1}+\sum_{j=2}^{N-1} \alpha_{j-1} p_{j},
$$

условие $\sigma_{0}\left(R_{k}\right)=\epsilon_{0}^{-k} R_{k}$ дает

$$
\alpha_{j-1}=\epsilon_{0}^{-k} \alpha_{j}, \quad j=2,3, \ldots, N-1, \quad \alpha_{N-1}=\epsilon_{0}^{-k} \alpha_{1},
$$

так что $\alpha_{j}=\epsilon_{0}^{j k} \alpha_{N-1}$ и

$$
R=\sum_{k=0}^{N-2} z^{k} f_{k}\left(z^{N-1}\right) \sum_{j=1}^{N-1} \epsilon_{0}^{j k} p_{j}+f_{N-1}\left(z^{N-1}\right) p_{N} .
$$

ПримеР 3.1. Для $N=3$

$$
R=f_{0}\left(z^{2}\right)\left(p_{1}+p_{2}\right)+z f_{1}\left(z^{2}\right)\left(p_{1}-p_{2}\right)+f_{2}\left(z^{2}\right) p_{3} .
$$

- Пусть $\sigma_{0}=\left(\begin{array}{ccccc}p_{1} & \cdots & p_{N-2} & p_{N-1} & p_{N} \\ p_{2} & \cdots & p_{1} & p_{N-1} & p_{N}\end{array}\right)$.

В этом случае $l_{0}=N-2,\left(\epsilon_{0}=e^{2 \pi i /(N-2)}\right)$. Заметим, что $p_{N-1}, p_{N} \in \mathbb{C}((k))$. Будем искать функции

$$
R_{k}=\sum_{j=1}^{N-2} \alpha_{j} p_{j} \quad \text { такие, что } \quad \sigma_{0}\left(R_{k}\right)=\epsilon_{0}^{-k} R_{k}, \quad k=0,1, \ldots, N-3 .
$$

Находим

$$
\begin{gathered}
\alpha_{j-1}=\epsilon_{0}^{-k} \alpha_{j}, \quad j=2,3, \ldots, N-2, \quad \alpha_{N-2}=\epsilon_{0}^{-k} \alpha_{1}, \\
\text { тогда } \alpha_{j}=\epsilon_{0}^{j k} \alpha_{N-2} \text { и } \\
R=\sum_{k=0}^{N-3} z^{k} f_{k}\left(z^{N-2}\right) \sum_{j=1}^{N-2} \epsilon_{0}^{j k} p_{j}+f_{N-2}\left(z^{N-2}\right) p_{N-1}+f_{N-1}\left(z^{N-2}\right) p_{N} .
\end{gathered}
$$

5 Теоретическая и математическая физика, т. 151, № 3, 2007 г. 
ПримеР 3.2. Для $N=4$

$$
R=f_{0}\left(z^{2}\right)\left(p_{1}+p_{2}\right)+z f_{1}\left(z^{2}\right)\left(p_{1}-p_{2}\right)+f_{2}\left(z^{2}\right) p_{3}+f_{3}\left(z^{2}\right) p_{4} .
$$

- Пусть $\sigma_{0}=\left(\begin{array}{ccccc}p_{1} & \cdots & p_{N-2} & p_{N-1} & p_{N} \\ p_{2} & \cdots & p_{1} & p_{N} & p_{N-1}\end{array}\right)$.

Как было указано в разделе 3 , показатель Ньютона для этого элемента $\sigma_{0}$ зависит от того, является ли $N$ четным или нечетным.

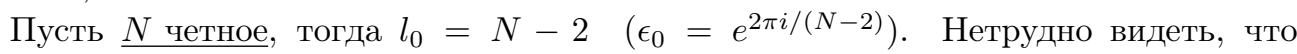
$p_{N-1}+p_{N} \in \mathbb{C}((k))$ и $\sigma_{0}\left(p_{N-1}-p_{N}\right)=-\left(p_{N-1}-p_{N}\right)$. С другой стороны, поскольку $\sigma_{0}$ действует на $p_{j}, j=1,2, \ldots, N-2$, так же, как и в предыдущем случае, и $\epsilon_{0}$ совпадает с предыдущим, снова имеем, что функции

$$
R_{k}=\sum_{j=1}^{N-2} \epsilon_{0}^{j k} p_{j}, \quad k=0,1, \ldots, N-3
$$

удовлетворяют условию $\sigma_{0}\left(R_{k}\right)=\epsilon_{0}^{-k} R_{k}$. Поэтому $R$ теперь дается выражением

$$
\begin{aligned}
R= & \sum_{k=0}^{N-3} z^{k} f_{k}\left(z^{N-2}\right) \sum_{j=1}^{N-2} \epsilon_{0}^{j k} p_{j}+ \\
& \quad+z^{(N-2) / 2} f_{N-2}\left(z^{N-2}\right)\left(p_{N-1}-p_{N}\right)+f_{N-1}\left(z^{N-2}\right)\left(p_{N-1}+p_{N}\right) .
\end{aligned}
$$

ПримеР 3.3. Для $N=4$

$$
R=f_{0}\left(z^{2}\right)\left(p_{1}+p_{2}\right)+z f_{1}\left(z^{2}\right)\left(p_{1}-p_{2}\right)+z f_{2}\left(z^{2}\right)\left(p_{3}-p_{4}\right)+f_{3}\left(z^{2}\right)\left(p_{3}+p_{4}\right) .
$$

Пусть $\underline{N \text { нечетное }}$, тогда $l_{0}=2(N-2) \quad\left(\epsilon_{0}=e^{\pi i /(N-2)}\right) . \quad$ В этом случае снова $p_{N-1}+p_{N} \in \mathbb{C}((k))$ и $\sigma_{0}\left(p_{N-1}-p_{N}\right)=-\left(p_{N-1}-p_{N}\right)$. Более того, если искать функции

$$
R_{k}=\sum_{j=1}^{N-2} \alpha_{j} p_{j} \quad \text { такие, что } \quad \sigma_{0}\left(R_{k}\right)=\epsilon_{0}^{-2 k} R_{k}, \quad k=0, \ldots, N-3,
$$

и действовать так же, как и ранее, то будем иметь $\alpha_{j}=\epsilon_{0}^{2 j k} \alpha_{N-2}$, так что

$$
\begin{aligned}
& R=\sum_{k=0}^{N-3} z^{2 k} f_{k}\left(z^{2(N-2)}\right) \sum_{j=1}^{N-2} \epsilon_{0}^{2 j k} p_{j}+ \\
& \quad+z^{N-2} f_{N-2}\left(z^{2(N-2)}\right)\left(p_{N-1}-p_{N}\right)+f_{N-1}\left(z^{2(N-2)}\right)\left(p_{N-1}+p_{N}\right) .
\end{aligned}
$$

ПримеР 3.4. Для $N=5$

$$
\begin{aligned}
R=f_{0} & \left(z^{6}\right)\left(p_{1}+p_{2}+p_{3}\right)+z^{2} f_{1}\left(z^{6}\right)\left(e^{2 \pi i / 3} p_{1}+e^{4 \pi i / 3} p_{2}+p_{3}\right)+ \\
& +z^{4} f_{2}\left(z^{6}\right)\left(e^{4 \pi i / 3} p_{1}+e^{2 \pi i / 3} p_{2}+p_{3}\right)+z^{3} f_{3}\left(z^{6}\right)\left(p_{4}-p_{5}\right)+f_{4}\left(z^{6}\right)\left(p_{4}+p_{5}\right) .
\end{aligned}
$$

Таким образом, интегрируемые деформации определяются выражениями (13)$(17)$ для функции $R$ в зависимости от элемента группы Галуа $\sigma_{0}$ и показателя Ньютона $l_{0}$. 


\section{4. НЕКОТОРЫЕ ПРИМЕРЫ И СООТВЕТСТВУЮЩИЕ ЗАКОНЫ СОХРАНЕНИЯ}

В этот раздел мы включили несколько примеров интегрируемых деформаций алгебраических кривых, соответствующих случаям $N=3$ и $N=4$. Прежде всего, отметим, что разложение компонент векторов $\mathbf{p}$ и $\mathbf{Q}$ в ряд по $z$ в первом уравнении для деформаций (2) приводит к бесконечному множеству законов сохранения. Именно в этом смысле мы называем деформации интегрируемыми. В приводимых примерах мы определим первые члены этих систем законов сохранения.

ПримеР 4.1. Пусть $N=3,\left(d_{1}, d_{2}, d_{3}\right)=(0,0,1)$.

Такой выбор степеней соответствует неприводимому случаю, и, следовательно, показатель Ньютона равен $l_{0}=3$. Из уравнений (4)-(6) находятся тривиальные уравнения $u_{10, t}=u_{31, t}=0$, поэтому берем

$$
u_{1}=1, \quad u_{2}=u_{20}, \quad u_{3}=k+u_{30},
$$

и, выбирая

$$
R=\frac{27}{4}(1-i \sqrt{3}) z^{5} \mathcal{L}_{2}
$$

получаем, что деформация задается следующей системой законов сохранения:

$$
\begin{aligned}
& u_{20, t}=\frac{5}{18} \partial_{x}\left(7 u_{20}+18 u_{20}^{2}+9 u_{20}^{3}+54 u_{20} u_{30}+12 u_{30}+81 u_{30}^{2}\right), \\
& u_{30, t}=\frac{5}{18} \partial_{x}\left(2 u_{20}^{2}+27 u_{30} u_{20}^{2}-u_{30}-27 u_{30}^{2}+6 u_{20}^{3}\right) .
\end{aligned}
$$

Эта система также может быть получена из уравнения (2). Действительно, если рассмотреть коэффициенты при $z^{-1}$ и $z^{-2}$ в (2), то мы придем к (18). С другой стороны, коэффициент при $z^{-3}$ дает тривиальные уравнения, в то время как из рассмотрения коэффициента при $z^{-4}$ мы получаем закон сохранения

$$
\begin{aligned}
\partial_{t}\left(5 u_{20}+\right. & \left.9 u_{20}^{2}+9 u_{30}+27 u_{20} u_{30}\right)= \\
=\partial_{x} & \left(\frac{175}{18} u_{20}+\frac{95}{2} u_{20}^{2}+\frac{195}{2} u_{20}^{3}+\frac{135}{2} u_{20}^{4}+\frac{85}{6} u_{30}+\frac{255}{2} u_{20} u_{30}+\frac{675}{2} u_{20}^{2} u_{30}+\right. \\
& \left.+\frac{675}{2} u_{20}^{2} u_{30}+\frac{405}{2} u_{20}^{3} u_{30}+90 u_{30}^{2}+405 u_{20} u_{30}^{2}+405 u_{30}^{3}\right) .
\end{aligned}
$$

Коэффициенты при $z^{-j}, j>4$, приводят к более сложным законам сохранения.

ПримеР 4.2. Пусть $N=3,\left(d_{1}, d_{2}, d_{3}\right)=(1,0,0)$.

Теперь показатель Ньютона равен $l_{0}=2$, и из уравнений (4)-(6) вытекает, что $\left(u_{30} / u_{11}\right)_{t}=0$. Поэтому полагая

$$
u_{1}=u_{11} k+u_{10}, \quad u_{2}=u_{20}, \quad u_{3}=u_{11}
$$

и выбирая $R$ в виде

$$
R=z^{4}\left(p_{1}+p_{2}\right)
$$


получаем

$$
\begin{aligned}
& u_{11, t}=\partial_{x}\left(-\frac{2 u_{10}}{u_{11}}\right), \quad u_{10, t}=\partial_{x}\left(-\frac{u_{10}^{2}+2 u_{20}}{u_{11}^{2}}\right), \\
& u_{20, t}=\frac{1}{u_{11}^{3}}\left[2 u_{10} u_{20} u_{11, x}+u_{11}\left(-2 u_{20} u_{10, x}+4 u_{11, x}\right)\right],
\end{aligned}
$$

где только первые два уравнения являются законами сохранения. Новые законы сохранения могут быть получены из уравнения (2). Например, рассматривая коэффициенты при $z^{-2}, z^{-3}$ и $z^{-4}$, получаем

$$
\begin{gathered}
\partial_{t}\left(\frac{u_{20}}{u_{11}}\right)= \\
\partial_{x}\left(-\frac{2}{u_{11}^{2}}\right), \quad \partial_{t}\left(\frac{4 u_{10}}{u_{11}}+\frac{u_{20}^{2}}{u_{11}^{2}}\right)=\partial_{x}\left(-\frac{8 u_{20}}{u_{11}^{3}}\right), \\
\partial_{t}\left(\frac{1}{u_{11}}-\frac{u_{10} u_{20}}{u_{11}^{2}}\right)=\partial_{x}\left(\frac{2 u_{10}}{u_{11}^{3}}+\frac{u_{20}^{2}}{u_{11}^{4}}\right) .
\end{gathered}
$$

ПримеР 4.3. Пусть $N=4,\left(d_{1}, d_{2}, d_{3}, d_{4}\right)=(0,1,0,0)$.

При таком выборе степеней показатель Ньютона равен $l_{0}=2$ и $\left(u_{40} / u_{21}\right)_{t}=0$. Поэтому положим

$$
u_{1}=u_{10}, \quad u_{2}=u_{21} k+u_{20}, \quad u_{3}=u_{30}, \quad u_{4}=u_{21} .
$$

Нумеруя ветви кривой $\mathcal{C}$ так, что $p_{1,2} \sim z+O(1), p_{3,4} \sim z^{-1}+O\left(z^{-2}\right)$, и выбирая

$$
R=z^{4}\left(p_{3}+p_{4}\right)
$$

получаем

$$
\begin{aligned}
u_{10, t}= & \partial_{x}\left(\frac{2 u_{10}^{2} u_{20}+2 u_{20}^{2}-4 u_{21}+2 u_{10} u_{30}}{u_{21}^{2}}\right), \\
u_{21, t}= & 4 u_{21}^{1 / 2} \partial_{x}\left(\frac{2 u_{10} u_{20}-2 u_{30}}{u_{21}^{3 / 2}}\right) \\
u_{20, t}= & u_{21}^{-2}\left[\left(4 u_{20}^{2}-2 u_{10} u_{30}+4 u_{21}\right) u_{10, x}+\left(4 u_{10} u_{20}+2 u_{30}\right) u_{20, x}-2 u_{10}^{2} u_{30, x}\right]+ \\
& \quad+u_{21}^{-3}\left(6 u_{10} u_{21}-6 u_{10} u_{20}^{2}+4 u_{10}^{2} u_{30}-2 u_{20} u_{30}-14 u_{10} u_{21}\right) u_{21, x}, \\
u_{30, t}= & u_{21}^{-2}\left[\left(4 u_{20} u_{30}-4 u_{10} u_{21}\right) u_{10, x}+\left(4 u_{10} u_{30}+4 u_{21}\right) u_{20, x}-4 u_{30} u_{30, x}\right]+ \\
& \quad+u_{21}^{-3}\left[\left(4 u_{20}-2 u_{10}^{2}-12 u_{20}\right) u_{21}-6 u_{10} u_{20} u_{30}+6 u_{30}^{2}+6 u_{10}^{2} u_{21}\right] u_{21, x} .
\end{aligned}
$$

Здесь первое уравнение сразу имеет вид закона сохранения, а второе может быть преобразовано к таком виду для $u_{21}^{1 / 2}$. Новые законы сохранения, соответствующие системе (20), могут быть выведены из уравнения (2). Так, из рассмотрения коэффициентов при $z^{-1}$ находим

$$
\partial_{t}\left(\frac{4 u_{10}^{2}+4 u_{20}}{u_{21}^{1 / 2}}\right)=\partial_{x}\left(\frac{2 u_{10}^{3} u_{20}+8 u_{10} u_{20}^{2}+16 u_{10} u_{21}-2 u_{10}^{2} u_{30}+8 u_{20} u_{30}}{u_{21}^{5 / 2}}\right)
$$


а коэффициентам при $z^{-j}, j=2,3,4$, для двух последних компонент уравнения (2) отвечают следующие законы сохранения:

$$
\begin{aligned}
\partial_{t}\left(\frac{u_{30}}{u_{21}}\right) & =\partial_{x}\left(\frac{4 u_{20}-2 u_{10}^{2}}{u_{21}^{2}}\right), \\
\partial_{t}\left(\frac{4 u_{20} u_{21}+u_{30}^{2}}{u_{21}^{2}}\right) & =\partial_{x}\left(\frac{16 u_{10} u_{21}-8 u_{10}^{2} u_{30}+16 u_{20} u_{30}}{u_{21}^{3}}\right), \\
\partial_{t}\left(\frac{u_{10} u_{21}+u_{20} u_{30}}{u_{21}^{2}}\right) & =\partial_{x}\left(-\frac{2}{u_{21}^{2}}+\frac{4 u_{20}^{2}-2 u_{10}^{2} u_{20}+6 u_{10} u_{30}}{u_{21}^{3}}+\frac{\left(2 u_{20}-u_{10}^{2}\right) u_{30}^{2}}{u_{21}^{4}}\right) .
\end{aligned}
$$

Благодарности. Авторы выражают благодарность Ю. Кодаме за его непрерывное внимание и помощь во время выполнения этой работы и М. Павлову за полезные советы. Работа была частично поддержана MEC (проект № FIS2005-00319) и грантом COFIN 2004 "Sintesi".

\section{Список литературы}

[1] В.Е. Захаров, С.В. Манаков, С. П. Новиков, Л.П. Питаевский, Теория солитонов. Метод обратной задачи, Наука, M., 1980; E. D. Belokolos, A. I. Bobenko, V. Z. Enol'ski, A.R. Its, V.B. Matveev, Algebro-Geometric approach to nonlinear integrable equations, Springer Ser. Nonlinear Dynamics, Springer, Berlin, 1994; Б. А. Дубровин, С. П. Новиков, УMH, 44:6 (1989), 29; H. Flaschka, M. G. Forest, D. W. Mclauglin, Commun. Pure Appl. Math., 33 (1980), 739; B. A. Dubrovin, Commun. Math. Phys., 145 (1992), 195.

[2] И. М. Кричевер, Функи. анализ и его прилож., 22:3 (1988), 37; I. M. Krichever, Commun. Pure Appl. Math., 47 (1994), 437.

[3] Y. Kodama, B. G. Konopelchenko, J. Phys. A, 35 (2002), L489.

[4] B. G. Konopelchenko, L. Martínez Alonso, J. Phys. A, 37 (2004), 7859.

[5] Ю. Кодама, Б. Г. Конопельченко, Л. Мартинес Алонсо, ТМФ, 144 (2005), 94.

[6] Y. Kodama, B. G. Konopelchenko, L. Martínez Alonso, E. Medina, J. Math. Phys., 46 (2005), 113502.

[7] B. G. Konopelchenko, L. Martínez Alonso, E. Medina, J. Phys. A, 39 (2006), 11231.

[8] C. L. Siegel, Topics in complex function theory, v. I. Elliptic Functions and Uniformization Theory, Wiley, New York-London-Sydney, 1969.

[9] R. Y. Walker, Algebraic Curves, Springer, New York-Heidelberg-Berlin, 1978; S. S. Abhyankar, Algebraic Geometry for Scientists and Engineers, Math. Surveys Monogr., 35, AMS, Providence, RI, 1990.

[10] L. Redei, Algebra, v. I, Pergamon, Oxford-New York-Toronto, 1967. 